Supporting Information (Cartesian coordinates and total energies of the optimized minima and transition structures)

\title{
The DMDO Hydroxylation of Hydrocarbons via the Oxygen Rebound
}

\section{Mechanism}

Robert D. Bach

Department of Chemistry and Biochemistry, University of Delaware, Newark, Delaware 19716.

Content

\section{Full reference $\mathbf{1 0}$}

2. Supporting Information

All the computational studies reported here were performed using the Gaussian 09 suite of programs. $^{1}$ GridChem, Computational Chemistry Grid, (www.gridchem.org) is acknowledged for computational resources and services for the minima and transition state reported in this publication.

CONTENT

\section{(1 Full Reference of 10}

Frisch, M. J. T., G. W.; Schlegel, H. B.; Scuseria, G. E.; Robb, M. A.; Cheeseman, J. R.; Scalmani, G.; Barone, V.; Mennucci, B.; Petersson, G. A.; Nakatsuji, H.; Caricato, M.; Li, X.; Hratchian, H. P.; Izmaylov, A. F.; Bloino, J.; Zheng, G.; Sonnenberg, J. L.; Hada, M.; Ehara, M.; Toyota, K.; Fukuda, R.; Hasegawa, J.; Ishida, M.; Nakajima, T.; Honda, Y.; Kitao, O.; Nakai, H.; Vreven, T.; Montgomery, Jr., J. A.; Peralta, J. E.; Ogliaro, F.; Bearpark, M.; Heyd, J. J.; Brothers, E.; Kudin, K. N.; Staroverov, V. N.; Kobayashi, R.; Normand, J.; Raghavachari, K.; Rendell, A.; Burant, J. C.; Iyengar, S. S.; Tomasi, J.; Cossi, M.; Rega, N.; Millam, N. J.; Klene, M.; Knox, J. E.; Cross, J. B.; Bakken, V.; Adamo, C.; Jaramillo, J.; Gomperts, R.; Stratmann, R. E.; Yazyev, O.; Austin, A. J.; Cammi, R.; Pomelli, C.; Ochterski, J. W.; Martin, R. L.; Morokuma, K.; Zakrzewski, V. G.; Voth, G. A.; Salvador, P.; Dannenberg, J. J.; Dapprich, S.; Daniels, A. D.; Farkas, Ö.; Foresman, J. B.; Ortiz, J. V.; Cioslowski, J.; Fox, D. J. ; B.01 ed.; Gaussian, Inc.: Wallingford CT, 2009. 
Summary of G4 calculations on peroxides presented in Table. All G4 calculations were initialized with fully optimized UM062X/6$311+\mathrm{G}(3 \mathrm{df}, 2 \mathrm{p})$ coordinates with a stable wavefunction (stable $=\mathrm{opt})$ as the initial guess (guess $=$ read).

\section{$\mathrm{HO}-\mathrm{OH}$}

G4 Enthalpy= $-151.490363$

$\mathrm{HO}$ radical

G4 Enthalpy= $\mathrm{MeO}-\mathrm{OH}$

G4 Enthalpy=

$\mathrm{MeO}$ radical

G4 Enthalpy=

$\mathrm{MeO}-\mathrm{OMe}$

G4 Enthalpy=

$\left(\mathrm{CH}_{3}\right)_{3} \mathrm{CO}-\mathrm{OH}$

G4 Enthalpy=

$(\mathrm{CH} 3)_{3} \mathrm{CO}$ radical

G4 Enthalpy=

$\mathrm{CH} 3(\mathrm{C}=\mathrm{O}) \mathrm{O}-\mathrm{OH}$

G4 Enthalpy=

$\mathrm{CH} 3(\mathrm{C}=\mathrm{O}) \mathrm{O}$ radical

$-75.706168$

$-190.757183$

$-114.982125$

$-230.024150$

$-308.605786$

$-232.829605$

G4 Enthalpy=

$(\mathrm{CH} 3)_{3} \mathrm{CO}-\mathrm{OC}(\mathrm{CH} 3)_{3}$

G4 Enthalpy=

$-304.082239$

$-228.321213$

$-465.722072$

Selected peroxides optimized with the G4 protocol.

\section{$\left(\mathrm{CH}_{3}\right)_{3} \mathrm{CO}-\mathrm{OH}$}

$\begin{array}{crrr}\text { scf } & \text { done: } \quad-307.056067 & \\ \text { C } & -0.269541 & 0.144568 & -1.567301 \\ \text { C } & -0.322554 & 0.198620 & -0.037456 \\ \text { H } & 0.765665 & 0.113757 & -1.913109 \\ \text { H } & -0.783136 & -0.748874 & -1.933558 \\ \text { H } & -0.754106 & 1.025701 & -1.999496 \\ \text { C } & -1.765165 & 0.122576 & 0.472887 \\ \text { C } & 0.395689 & 1.436839 & 0.509665 \\ \text { H } & -1.791509 & 0.131091 & 1.566151\end{array}$




$\begin{array}{rrrr}\mathrm{H} & -2.337003 & 0.979235 & 0.105244 \\ \mathrm{H} & -2.247937 & -0.793444 & 0.122056 \\ \mathrm{H} & -0.083383 & 2.351302 & 0.147191 \\ \mathrm{H} & 0.365617 & 1.446461 & 1.604037 \\ \mathrm{H} & 1.439304 & 1.451478 & 0.185984 \\ \mathrm{O} & 0.250557 & -1.004015 & 0.522194 \\ \mathrm{O} & 1.632759 & -1.115244 & 0.109743 \\ \mathrm{H} & 2.082303 & -0.916507 & 0.942693\end{array}$

\section{$(\mathrm{CH} 3)_{3} \mathrm{CO}$ radical}

$\begin{array}{crrr}\text { scf done: } & -233.003555 & \\ \text { C } & -0.389179 & 1.359854 & 0.719062 \\ \text { C } & -0.387793 & 0.001035 & 0.037890 \\ \text { H } & 0.510498 & 1.483185 & 1.324279 \\ \mathrm{H} & -0.415219 & 2.152100 & -0.033299 \\ \mathrm{H} & -1.264913 & 1.459575 & 1.367373 \\ \mathrm{C} & -1.584842 & -0.157806 & -0.888159 \\ \mathrm{C} & -0.308528 & -1.138323 & 1.041945 \\ \mathrm{H} & -1.546717 & -1.121660 & -1.402210 \\ \mathrm{H} & -2.510748 & -0.105919 & -0.309068 \\ \mathrm{H} & -1.593346 & 0.638635 & -1.636115 \\ \mathrm{H} & -1.189143 & -1.137566 & 1.690646 \\ \mathrm{H} & -0.265590 & -2.097602 & 0.517735 \\ \mathrm{H} & 0.579713 & -1.034497 & 1.667977 \\ \mathrm{O} & 0.709707 & -0.078799 & -0.899589\end{array}$

\section{$(\mathrm{CH} 3)_{3} \mathrm{CO}-\mathrm{OC}(\mathrm{CH} 3)_{3}$}

$\begin{array}{crrr}\text { sCf } & \text { done }: & -463.258148 & \\ \mathrm{C} & -1.671639 & 0.488249 & -0.880153 \\ \mathrm{C} & 1.599715 & -0.538985 & 0.095406 \\ \mathrm{C} & 2.088823 & -1.989830 & 0.171157 \\ \mathrm{H} & 3.168588 & -2.009981 & 0.343994 \\ \mathrm{H} & 1.596380 & -2.521231 & 0.990110 \\ \mathrm{H} & 1.877995 & -2.518971 & -0.762453 \\ \mathrm{C} & 1.846392 & 0.193209 & 1.420837 \\ \mathrm{H} & 1.451190 & 1.209962 & 1.380548 \\ \mathrm{H} & 1.354784 & -0.335414 & 2.242620 \\ \mathrm{H} & 2.918790 & 0.245872 & 1.633781 \\ \mathrm{C} & 2.244563 & 0.199112 & -1.083922 \\ \mathrm{H} & 3.327365 & 0.272551 & -0.941704 \\ \mathrm{H} & 2.055587 & -0.336286 & -2.019142 \\ \mathrm{H} & 1.837856 & 1.207836 & -1.173912\end{array}$




$\begin{array}{lrrr}\mathrm{C} & -2.197670 & 1.927856 & -0.852047 \\ \mathrm{H} & -3.170803 & 1.977835 & -1.348588 \\ \mathrm{H} & -2.314846 & 2.276364 & 0.177696 \\ \mathrm{H} & -1.509485 & 2.601300 & -1.370615 \\ \mathrm{C} & -1.453421 & 0.009219 & -2.320358 \\ \mathrm{H} & -0.788649 & 0.694556 & -2.854569 \\ \mathrm{H} & -1.005389 & -0.985907 & -2.329345 \\ \mathrm{H} & -2.406613 & -0.033991 & -2.856532 \\ \mathrm{C} & -2.606440 & -0.452921 & -0.109242 \\ \mathrm{H} & -2.729981 & -0.104387 & 0.920197 \\ \mathrm{H} & -3.591554 & -0.486873 & -0.585183 \\ \mathrm{H} & -2.200061 & -1.465732 & -0.082773 \\ \mathrm{O} & 0.189497 & -0.711880 & -0.121616 \\ \mathrm{O} & -0.416920 & 0.616550 & -0.190598\end{array}$

All the structures presented in the Figures were fully optimized at the UM062X/6-311+G(3df,2p) level unless noted otherwise. The G4 energies are derived from a stable wavefunction as noted above.

\section{Figure 1 \\ DMDO and its TS for O-O bond homolysis to its diradical minimum. \\ DMDO}

scf done: $\quad-268.250451$

Frequencies --

$\mathrm{C}$

O

O

C

C

$\mathrm{H}$

$\mathrm{H}$

$\mathrm{H}$

$\mathrm{H}$

$\mathrm{H}$

$\mathrm{H}$

G4 Energy=

G4 Free Energy=
162.2841

$$
\begin{array}{rr}
0.000000 & -0.096442 \\
0.000000 & 1.081034 \\
0.000000 & 1.081034 \\
1.284940 & -0.870877 \\
-1.284940 & -0.870877 \\
2.122978 & -0.178874 \\
-2.122978 & -0.178874 \\
1.335202 & -1.507567 \\
-1.335202 & -1.507567 \\
1.335202 & -1.507567 \\
-1.335202 & -1.507567
\end{array}
$$




\section{DMDO-TS}

S2=0.860671 Frequencies -scf done: -268.202192

$\begin{array}{rrrr}\mathrm{C} & -0.002787 & -0.012121 & 0.003646 \\ \mathrm{O} & 0.120030 & -0.994258 & 0.986786 \\ \mathrm{O} & -0.070566 & -0.954898 & -1.022437 \\ \mathrm{C} & -1.298003 & 0.772587 & 0.090135 \\ \mathrm{C} & 1.245435 & 0.848296 & -0.046103 \\ \mathrm{H} & 1.186067 & 1.517391 & -0.904591 \\ \mathrm{H} & 1.330614 & 1.449223 & 0.859167 \\ \mathrm{H} & 2.119676 & 0.209086 & -0.143418 \\ \mathrm{H} & -1.275793 & 1.405112 & 0.977621 \\ \mathrm{H} & -1.418543 & 1.407358 & -0.787601 \\ \mathrm{H} & -2.134209 & 0.080825 & 0.157829\end{array}$

G4 Energy=

G4 Free Energy=

\section{DMDO-Diradical}

S2 $=0.957856$ Frequencies -scf done: -268.219551

$\begin{array}{lrr}\mathrm{C} & 0.000000 & -0.142951 \\ \mathrm{O} & -0.000057 & -0.858843 \\ \mathrm{O} & 0.000057 & -0.858843 \\ \mathrm{C} & 1.279898 & 0.737886 \\ \mathrm{C} & -1.279898 & 0.737886 \\ \mathrm{H} & -1.274761 & 1.354616 \\ \mathrm{H} & -1.274836 & 1.354636 \\ \mathrm{H} & -2.158769 & 0.097624 \\ \mathrm{H} & 1.274761 & 1.354616 \\ \mathrm{H} & 1.274836 & 1.354635 \\ \mathrm{H} & 2.158769 & 0.097625\end{array}$

G4 Energy=

G4 Free Energy=
$-529.5200$

$-268.078199$

$-268.112397$

$$
217.6760
$$

0.000000
-1.150551
1.150550
-0.000065
0.000065
0.894786
-0.894642
0.000095
-0.894786
0.894643
-0.000095

Figure 2. Ground State (GS) Complex between DMDO and isobutane and TS-2 for 0-O bond homolysis to Diradical Minimum-3 and its TS-4 for 1,2methyl migration.

\section{GS-1}

S2=0 Frequencies -- $\quad 21.3284$ 


$\begin{array}{crrr}\text { sCf } & \text { done: } & -426.669800 & \\ \mathrm{O} & 2.728073 & 0.311551 & -1.122028 \\ \mathrm{O} & 1.296624 & 0.352563 & -0.823187 \\ \mathrm{C} & 2.228097 & -0.076783 & 0.111323 \\ \mathrm{C} & -1.852387 & 0.428288 & -0.245381 \\ \mathrm{H} & -1.172121 & 1.070741 & -0.810859 \\ \mathrm{C} & -1.950505 & -0.910037 & -0.970259 \\ \mathrm{C} & -1.272922 & 0.235455 & 1.152553 \\ \mathrm{C} & -3.215499 & 1.109103 & -0.169147 \\ \mathrm{C} & 2.491631 & 0.858374 & 1.254346 \\ \mathrm{C} & 2.248540 & -1.551311 & 0.389663 \\ \mathrm{H} & -2.596060 & -1.596699 & -0.415849 \\ \mathrm{H} & -2.370555 & -0.788504 & -1.969717 \\ \mathrm{H} & -0.966481 & -1.370473 & -1.071453 \\ \mathrm{H} & -3.919721 & 0.485915 & 0.388406 \\ \mathrm{H} & -3.149732 & 2.073748 & 0.336651 \\ \mathrm{H} & -3.631204 & 1.275530 & -1.163811 \\ \mathrm{H} & -1.949437 & -0.366067 & 1.765601 \\ \mathrm{H} & -0.315278 & -0.285533 & 1.108556 \\ \mathrm{H} & -1.119328 & 1.190883 & 1.657387 \\ \mathrm{H} & 3.478484 & 0.664337 & 1.675022 \\ \mathrm{H} & 2.441448 & 1.884109 & 0.897957 \\ \mathrm{H} & 1.748377 & 0.707228 & 2.037225 \\ \mathrm{H} & 3.235366 & -1.847059 & 0.746188 \\ \mathrm{H} & 1.519354 & -1.798895 & 1.161399 \\ \mathrm{H} & 2.013110 & -2.093012 & -0.523029\end{array}$

G4 Energy=

G4 Free Energy=
$-426.415393$

$-426.474584$

\section{TS-2}

S2=0.972321 Frequencies -scf done: -426.629397

$\begin{array}{rrrr}\mathrm{O} & 2.294661 & 0.914261 & -2.552598 \\ \mathrm{O} & 0.583181 & -0.613305 & -2.382251 \\ \mathrm{C} & 1.250235 & 0.455327 & -1.824991 \\ \mathrm{C} & 0.057796 & 0.001432 & -4.839688 \\ \mathrm{H} & 0.197877 & -0.156649 & -3.680060 \\ \mathrm{C} & 1.239428 & -0.729485 & -5.445047 \\ \mathrm{C} & -1.272683 & -0.651637 & -5.160468 \\ \mathrm{C} & 0.078075 & 1.488409 & -5.137293 \\ \mathrm{C} & 0.313198 & 1.650766 & -1.548553 \\ \mathrm{C} & 1.823202 & -0.101413 & -0.491508 \\ \mathrm{H} & 1.187829 & -0.679832 & -6.537398 \\ \mathrm{H} & 2.178478 & -0.281755 & -5.120923\end{array}$




$\begin{array}{rrrr}\mathrm{H} & 1.240077 & -1.779083 & -5.150709 \\ \mathrm{H} & 0.016126 & 1.642272 & -6.219427 \\ \mathrm{H} & -0.770191 & 2.003370 & -4.684322 \\ \mathrm{H} & 1.002147 & 1.949604 & -4.785748 \\ \mathrm{H} & -1.456704 & -0.607612 & -6.238702 \\ \mathrm{H} & -1.276826 & -1.699724 & -4.860651 \\ \mathrm{H} & -2.096949 & -0.144767 & -4.657842 \\ \mathrm{H} & 0.846099 & 2.438649 & -1.021835 \\ \mathrm{H} & -0.066193 & 2.039765 & -2.490920 \\ \mathrm{H} & -0.520254 & 1.290532 & -0.948779 \\ \mathrm{H} & 2.342704 & 0.702701 & 0.023985 \\ \mathrm{H} & 0.997908 & -0.470540 & 0.112305 \\ \mathrm{H} & 2.511375 & -0.913747 & -0.709540\end{array}$

G4 Energy=

G4 Free Energy=

$-426.370358$

$-426.427862$

\section{Diradical Min-3}

\begin{tabular}{crrr} 
S2 $=0$ & Frequencies & \multicolumn{1}{c}{12.6490} \\
SCf & done: -426.639899 & \\
$\mathrm{O}$ & -1.084606 & -0.597928 & -0.942666 \\
$\mathrm{O}$ & -1.048714 & -0.025335 & 1.284698 \\
$\mathrm{C}$ & -1.729071 & -0.042124 & 0.112459 \\
$\mathrm{C}$ & 2.132041 & -0.207041 & 0.093030 \\
$\mathrm{H}$ & 1.310402 & -0.890544 & 0.318032 \\
$\mathrm{C}$ & 3.039024 & -0.120867 & 1.316156 \\
$\mathrm{C}$ & 2.890345 & -0.745616 & -1.115441 \\
$\mathrm{C}$ & 1.525820 & 1.156220 & -0.221200 \\
$\mathrm{C}$ & -2.986226 & -0.933674 & 0.363920 \\
$\mathrm{C}$ & -2.151610 & 1.401993 & -0.252090 \\
$\mathrm{H}$ & 3.861819 & 0.575858 & 1.133934 \\
$\mathrm{H}$ & 2.487440 & 0.230358 & 2.189716 \\
$\mathrm{H}$ & 3.471610 & -1.093175 & 1.557057 \\
$\mathrm{H}$ & 2.311434 & 1.885686 & -0.437691 \\
$\mathrm{H}$ & 0.872537 & 1.099036 & -1.094897 \\
$\mathrm{H}$ & 0.944623 & 1.529954 & 0.625103 \\
$\mathrm{H}$ & 3.709871 & -0.073663 & -1.384716 \\
$\mathrm{H}$ & 3.318164 & -1.728040 & -0.909595 \\
$\mathrm{H}$ & 2.231513 & -0.837315 & -1.980405 \\
$\mathrm{H}$ & -3.578773 & -0.949621 & -0.546668 \\
$\mathrm{H}$ & -2.669942 & -1.941380 & 0.620304 \\
$\mathrm{H}$ & -3.549348 & -0.499722 & 1.185519 \\
$\mathrm{H}$ & -2.753621 & 1.373007 & -1.156684 \\
$\mathrm{H}$ & -2.720995 & 1.818760 & 0.574778 \\
$\mathrm{H}$ & -1.262797 & 2.004149 & -0.423790
\end{tabular}


G4 Energy=

G4 Free Energy=

Rearrangement-TS-4

S2 $=0.57867$ Frequencies scf done: -426.634944

$\begin{array}{rrrr}\mathrm{O} & -1.079357 & -0.642313 & -0.623948 \\ \mathrm{O} & -0.771713 & 0.089023 & 1.504204 \\ \mathrm{C} & -1.378987 & 0.143865 & 0.361053 \\ \mathrm{C} & 2.189352 & -0.158897 & -0.009321 \\ \mathrm{H} & 1.375488 & -0.814567 & 0.308089 \\ \mathrm{C} & 3.148899 & 0.019987 & 1.162277 \\ \mathrm{C} & 2.891240 & -0.801488 & -1.201009 \\ \mathrm{C} & 1.577417 & 1.180321 & -0.407951 \\ \mathrm{C} & -2.660940 & -0.949031 & 0.922224 \\ \mathrm{C} & -2.044239 & 1.476681 & -0.001168 \\ \mathrm{H} & 3.972209 & 0.684418 & 0.885678 \\ \mathrm{H} & 2.638201 & 0.454915 & 2.022834 \\ \mathrm{H} & 3.579145 & -0.934210 & 1.470692 \\ \mathrm{H} & 2.354370 & 1.873974 & -0.741722 \\ \mathrm{H} & 0.865987 & 1.055505 & -1.227697 \\ \mathrm{H} & 1.061386 & 1.639672 & 0.438551 \\ \mathrm{H} & 3.702347 & -0.161057 & -1.558303 \\ \mathrm{H} & 3.321405 & -1.767728 & -0.932905 \\ \mathrm{H} & 2.195051 & -0.957777 & -2.026456 \\ \mathrm{H} & -3.310231 & -0.992980 & 0.058302 \\ \mathrm{H} & -2.181898 & -1.883767 & 1.173287 \\ \mathrm{H} & -3.063176 & -0.407398 & 1.767757 \\ \mathrm{H} & -2.769453 & 1.335172 & -0.798019 \\ \mathrm{H} & -2.508003 & 1.918959 & 0.876478 \\ \mathrm{H} & -1.257226 & 2.140763 & -0.356209\end{array}$

Figure 3. Hydrogen abstraction TS in a perpendicular orientation, Perp-TS-

5, concerted Oxygen Insertion TS-6 and rate-limiting H-Atom Abstraction

\section{Collinear TS-7.}

\section{Perp-TS-5}

S2=0.972321 Erequencies - - $\quad-771.0252$ scf done: -426.629397

$\begin{array}{rrrr}\mathrm{O} & 2.294661 & 0.914261 & -2.552598 \\ \mathrm{O} & 0.583181 & -0.613305 & -2.382251 \\ \mathrm{C} & 1.250235 & 0.455327 & -1.824991\end{array}$

$-426.382440$

$-426.440873$

$-429.4671$ 


$\begin{array}{lrrr}\mathrm{C} & 0.057796 & 0.001432 & -4.839688 \\ \mathrm{H} & 0.197877 & -0.156649 & -3.680060 \\ \mathrm{C} & 1.239428 & -0.729485 & -5.445047 \\ \mathrm{C} & -1.272683 & -0.651637 & -5.160468 \\ \mathrm{C} & 0.078075 & 1.488409 & -5.137293 \\ \mathrm{C} & 0.313198 & 1.650766 & -1.548553 \\ \mathrm{C} & 1.823202 & -0.101413 & -0.491508 \\ \mathrm{H} & 1.187829 & -0.679832 & -6.537398 \\ \mathrm{H} & 2.178478 & -0.281755 & -5.120923 \\ \mathrm{H} & 1.240077 & -1.779083 & -5.150709 \\ \mathrm{H} & 0.016126 & 1.642272 & -6.219427 \\ \mathrm{H} & -0.770191 & 2.003370 & -4.684322 \\ \mathrm{H} & 1.002147 & 1.949604 & -4.785748 \\ \mathrm{H} & -1.456704 & -0.607612 & -6.238702 \\ \mathrm{H} & -1.276826 & -1.699724 & -4.860651 \\ \mathrm{H} & -2.096949 & -0.144767 & -4.657842 \\ \mathrm{H} & 0.846099 & 2.438649 & -1.021835 \\ \mathrm{H} & -0.066193 & 2.039765 & -2.490920 \\ \mathrm{H} & -0.520254 & 1.290532 & -0.948779 \\ \mathrm{H} & 2.342704 & 0.702701 & 0.023985 \\ \mathrm{H} & 0.997908 & -0.470540 & 0.112305 \\ \mathrm{H} & 2.511375 & -0.913747 & -0.709540 \\ & & & \end{array}$

G4 Energy=

G4 Free Energy=
$-426.377966$

$-426.429639$

\section{Conc-TS- 6}

S2=0 Frequencies - - $\quad-534.0343$

scf done: $\quad-426.606669$

$\begin{array}{rrrr}\mathrm{O} & 2.430015 & 0.231158 & -1.153082 \\ \mathrm{O} & 0.463268 & 0.056514 & -0.866274 \\ \mathrm{C} & 1.723578 & 0.039278 & -0.074610 \\ \mathrm{C} & -1.728581 & -0.004661 & 0.000762 \\ \mathrm{H} & -0.421542 & -0.210116 & -0.283126 \\ \mathrm{C} & -2.375549 & -0.641197 & -1.189922 \\ \mathrm{C} & -1.776858 & -0.777376 & 1.287097 \\ \mathrm{C} & -1.863303 & 1.479869 & 0.116337 \\ \mathrm{C} & 1.700226 & 1.192767 & 0.922280 \\ \mathrm{C} & 1.875744 & -1.325133 & 0.590159 \\ \mathrm{H} & -3.463522 & -0.582318 & -1.064578 \\ \mathrm{H} & -2.108683 & -0.116355 & -2.105033 \\ \mathrm{H} & -2.101701 & -1.690503 & -1.283806 \\ \mathrm{H} & -2.895864 & 1.711708 & 0.403586 \\ \mathrm{H} & -1.201563 & 1.882620 & 0.881542 \\ \mathrm{H} & -1.650363 & 1.967368 & -0.832398 \\ \mathrm{H} & -2.801641 & -0.727983 & 1.675180\end{array}$




$\begin{array}{rrrr}\mathrm{H} & -1.523494 & -1.826280 & 1.139664 \\ \mathrm{H} & -1.117945 & -0.344962 & 2.040087 \\ \mathrm{H} & 2.674271 & 1.268914 & 1.406228 \\ \mathrm{H} & 1.500556 & 2.117147 & 0.383276 \\ \mathrm{H} & 0.944829 & 1.044059 & 1.699038 \\ \mathrm{H} & 2.855082 & -1.384097 & 1.065455 \\ \mathrm{H} & 1.114141 & -1.492811 & 1.355880 \\ \mathrm{H} & 1.807466 & -2.095959 & -0.175048\end{array}$

G4 Energy=

G4 Free Energy=

$-426.388984$

$-426.441011$

\section{Coll-TS-7}

S2=0.530594 Frequencies -scf done: -426.618754

$\begin{array}{lrrr}\mathrm{O} & 2.506580 & 0.022063 & -1.159245 \\ \mathrm{O} & 0.640169 & -0.108589 & -0.986753 \\ \mathrm{C} & 1.695800 & -0.012632 & -0.059541 \\ \mathrm{C} & -1.669196 & -0.032385 & -0.011851 \\ \mathrm{H} & -0.519120 & -0.110266 & -0.389763 \\ \mathrm{C} & -2.429158 & -0.811918 & -1.061569 \\ \mathrm{C} & -1.712536 & -0.657742 & 1.363834 \\ \mathrm{C} & -1.966329 & 1.450168 & -0.021404 \\ \mathrm{C} & 1.668441 & 1.278789 & 0.734077 \\ \mathrm{C} & 1.863055 & -1.258289 & 0.787483 \\ \mathrm{H} & -3.499997 & -0.785112 & -0.835055 \\ \mathrm{H} & -2.280998 & -0.381635 & -2.051614 \\ \mathrm{H} & -2.113334 & -1.854517 & -1.085771 \\ \mathrm{H} & -3.012845 & 1.616021 & 0.254306 \\ \mathrm{H} & -1.342593 & 1.987905 & 0.692719 \\ \mathrm{H} & -1.804837 & 1.875758 & -1.011386 \\ \mathrm{H} & -2.737784 & -0.618945 & 1.746592 \\ \mathrm{H} & -1.405484 & -1.703415 & 1.337469 \\ \mathrm{H} & -1.077524 & -0.121343 & 2.070428 \\ \mathrm{H} & 2.623949 & 1.421507 & 1.239275 \\ \mathrm{H} & 1.492741 & 2.109912 & 0.054188 \\ \mathrm{H} & 0.882520 & 1.249640 & 1.490929 \\ \mathrm{H} & 2.819276 & -1.221843 & 1.310218 \\ \mathrm{H} & 1.068741 & -1.331630 & 1.531093 \\ \mathrm{H} & 1.841747 & -2.132194 & 0.139943\end{array}$

G4 Energy=

$-426.374699$

G4 Free Energy=

$-426.426517$ 
Figure 4. Rebound TS-8, Diradical TS-9. Final Product-10 and Minisci

\section{Hemiketal 11.}

\section{Reb-TS-8}

\begin{tabular}{|c|c|c|c|}
\hline & $\begin{array}{l}.696771 \text { Freo } \\
\text { done: }\end{array}$ & $\begin{array}{l}\text { cies -- } \\
785\end{array}$ & 55 \\
\hline 0 & 1.491814 & -0.660422 & 0.928401 \\
\hline 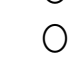 & -0.761778 & -0.392232 & 0.830210 \\
\hline & -3.093241 & 0.402796 & 0.860725 \\
\hline c & 0.536801 & -0.041787 & 0.259381 \\
\hline & -0.550539 & -0.803599 & 1.676026 \\
\hline & 0.49 & -0.568231 & -1.191638 \\
\hline C & 0.66 & 1.486092 & 0.289431 \\
\hline F & 0.413515 & -1.651929 & -1.169046 \\
\hline $\mathrm{t}$ & -0.381396 & -0.140451 & -1.681554 \\
\hline & 1.39 & -0.273875 & $-1 \cdot 7$ \\
\hline & 0.6 & 1.825516 & 1.324395 \\
\hline $\mathrm{H}$ & 1.61 & 1.772681 & -0.152423 \\
\hline $\mathrm{H}$ & -0.14 & 1.960249 & -0.263617 \\
\hline$\checkmark$ & -2.89 & 1.618818 & 1.685680 \\
\hline 11 & -2.63 & 1.371812 & 2.714938 \\
\hline $\mathrm{H}$ & -2.11 & $2.2 !$ & 1.2 \\
\hline 11 & -3.81 & 2.219315 & 1.71 \\
\hline $\mathrm{C}$ & -3.62 & -0.835137 & 1.476020 \\
\hline $\mathrm{H}$ & -4.72 & -0.838162 & 1.448934 \\
\hline $\mathrm{H}$ & -3. & $-1 \cdot 7$ & 0.929363 \\
\hline $\mathrm{H}$ & -3. & -0.9 & 2.521188 \\
\hline & $-3 \cdot 1$ & 0.5 & -0.614731 \\
\hline $\mathrm{H}$ & -2.84 & -0.4 & -1.090148 \\
\hline 11 & -4.121694 & 0.796937 & -0.968434 \\
\hline & -2.432 & 1.302466 & 6092 \\
\hline & 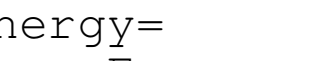 & & .390650 \\
\hline & 7 & & .447564 \\
\hline
\end{tabular}

\section{Dirad-Min-9}

S2=1.009239 Frequencies -scf done: -426.658445

24.1387

$\begin{array}{rrrr}\mathrm{O} & 2.990228 & 0.176911 & -0.800300 \\ \mathrm{O} & 0.795295 & 0.268737 & -1.309432 \\ \mathrm{C} & 1.753456 & 0.070304 & -0.291335 \\ \mathrm{C} & -1.994456 & 0.005877 & 0.333827 \\ \mathrm{H} & -0.079639 & 0.175183 & -0.916304 \\ \mathrm{C} & -2.378477 & -1.006053 & -0.695715 \\ \mathrm{C} & -2.026246 & -0.410229 & 1.766303\end{array}$




$\begin{array}{lrrr}\mathrm{C} & -2.226782 & 1.447864 & 0.021287 \\ \mathrm{C} & 1.603285 & 1.149088 & 0.800629 \\ \mathrm{C} & 1.612423 & -1.344984 & 0.304587 \\ \mathrm{H} & -3.465737 & -1.170807 & -0.694124 \\ \mathrm{H} & -2.109504 & -0.685891 & -1.704186 \\ \mathrm{H} & -1.910801 & -1.973617 & -0.504936 \\ \mathrm{H} & -3.296670 & 1.693102 & 0.088114 \\ \mathrm{H} & -1.705981 & 2.101884 & 0.722556 \\ \mathrm{H} & -1.906976 & 1.703762 & -0.990674 \\ \mathrm{H} & -3.058039 & -0.424703 & 2.146412 \\ \mathrm{H} & -1.624386 & -1.415174 & 1.906096 \\ \mathrm{H} & -1.467226 & 0.280055 & 2.400978 \\ \mathrm{H} & 2.337759 & 0.997802 & 1.588149 \\ \mathrm{H} & 1.737924 & 2.130847 & 0.351381 \\ \mathrm{H} & 0.597242 & 1.078457 & 1.215960 \\ \mathrm{H} & 2.345678 & -1.501626 & 1.092197 \\ \mathrm{H} & 0.606237 & -1.446987 & 0.713496 \\ \mathrm{H} & 1.755277 & -2.079192 & -0.485509 \\ & & & \end{array}$

\begin{tabular}{|c|c|c|c|}
\hline \\
\hline \multicolumn{4}{|c|}{$\begin{array}{l}\text { Prod-10 } \\
\text { S2 } 2=0 \text { Frequencies -- }\end{array}$} \\
\hline \multicolumn{4}{|c|}{ scf done: $\quad-426.7$} \\
\hline \multicolumn{4}{|c|}{-1.079562} \\
\hline \multicolumn{4}{|c|}{1.200410} \\
\hline $\mathrm{C}$ & -2.041883 & 0.714510 & -0.427353 \\
\hline \multicolumn{4}{|c|}{-0.178734} \\
\hline \multicolumn{4}{|r|}{-1.225737} \\
\hline \multicolumn{4}{|c|}{96795} \\
\hline \multicolumn{4}{|c|}{972} \\
\hline \multicolumn{4}{|r|}{187496} \\
\hline \multicolumn{4}{|c|}{1.404750} \\
\hline \multicolumn{4}{|r|}{160} \\
\hline \multicolumn{4}{|c|}{-0.289221} \\
\hline \multicolumn{4}{|l|}{$\mathrm{H}$} \\
\hline \multicolumn{4}{|c|}{0.881376} \\
\hline \multicolumn{4}{|c|}{3.307435} \\
\hline $\mathrm{H}$ & 3.141970 & 1.406764 & -1.128422 \\
\hline $\mathrm{H}$ & 1.800919 & 1.966727 & -0.117255 \\
\hline $\mathrm{H}$ & 3.902138 & -1.102860 & 0.526030 \\
\hline $\mathrm{H}$ & 2.773744 & -2.207182 & -0.281697 \\
\hline \multirow{2}{*}{$\begin{array}{l}\mathrm{H} \\
\mathrm{H}\end{array}$} & 3.702183 & -1.043477 & -1.235501 \\
\hline & -3.687043 & 1.095455 & 0.894052 \\
\hline
\end{tabular}




$\begin{array}{rrrr}\mathrm{H} & -3.212776 & 2.482645 & -0.129721 \\ \mathrm{H} & -4.101587 & 1.098074 & -0.810899 \\ \mathrm{H} & -2.878502 & -1.234601 & -0.738993 \\ \mathrm{H} & -1.087776 & -1.196055 & -0.663546 \\ \mathrm{H} & -2.073105 & -1.015490 & 0.805703\end{array}$

G4 Energy=

$-426.533059$

G4 Free Energy=

$-426.588581$

\section{Hemiketal-11}

S2=0 Frequencies -- 80.6109 scf done: -426.789528

$\begin{array}{rrrr}\mathrm{O} & -1.285791 & -0.401598 & 0.520027 \\ \mathrm{O} & -0.799857 & 0.162224 & -1.681586 \\ \mathrm{C} & -2.464612 & 0.316045 & 0.915762 \\ \mathrm{C} & -0.304592 & 0.091446 & -0.361309 \\ \mathrm{H} & -1.109678 & -0.715188 & -1.923857 \\ \mathrm{C} & 0.815338 & -0.938091 & -0.275590 \\ \mathrm{C} & 0.218918 & 1.484040 & -0.041623 \\ \mathrm{H} & 0.415184 & -1.927913 & -0.498337 \\ \mathrm{H} & 1.596511 & -0.695145 & -0.993740 \\ \mathrm{H} & 1.236043 & -0.958755 & 0.728429 \\ \mathrm{H} & -0.538656 & 2.242515 & -0.220845 \\ \mathrm{H} & 0.546866 & 1.533874 & 0.995101 \\ \mathrm{H} & 1.065371 & 1.695228 & -0.693249 \\ \mathrm{C} & -2.148864 & 1.327938 & 2.020169 \\ \mathrm{H} & -1.506079 & 0.865860 & 2.770166 \\ \mathrm{H} & -1.659928 & 2.222098 & 1.641105 \\ \mathrm{H} & -3.075621 & 1.637321 & 2.505152 \\ \mathrm{C} & -3.348479 & -0.785332 & 1.495822 \\ \mathrm{H} & -4.293198 & -0.372593 & 1.850080 \\ \mathrm{H} & -3.554683 & -1.536520 & 0.733694 \\ \mathrm{H} & -2.841380 & -1.271043 & 2.329661 \\ \mathrm{C} & -3.192409 & 0.987507 & -0.246363 \\ \mathrm{H} & -3.444151 & 0.258674 & -1.015762 \\ \mathrm{H} & -4.116693 & 1.428276 & 0.130804 \\ \mathrm{H} & -2.599944 & 1.772886 & -0.709021\end{array}$

G4 Energy=

$-426.530191$

G4 Free Energy=

$-426.577743$ 
Figure 5. Epoxidation TS for DMDO and Ethylene with the UM062X and B3LYP DFT Functionals and the O-O bond Homolysis TS Complexed to Ethylene.

\section{Epox-TS-12a}

S2=0 Frequencies - $\quad-727.6869$

$\begin{array}{rrrr}\text { SCf } & \text { done: } & -346.774611 & \\ \mathrm{C} & -0.824685 & 0.041698 & 0.334206 \\ \mathrm{O} & -0.848504 & 0.099986 & 1.645336 \\ \mathrm{O} & 0.633104 & -0.006106 & 0.574994 \\ \mathrm{C} & -1.233910 & 1.301304 & -0.407396 \\ \mathrm{C} & -1.336968 & -1.241568 & -0.294295 \\ \mathrm{H} & -0.747107 & 2.160201 & 0.050200 \\ \mathrm{H} & -0.907900 & -2.093262 & 0.229774 \\ \mathrm{H} & -0.991512 & 1.251567 & -1.469979 \\ \mathrm{H} & -2.313955 & 1.418563 & -0.314723 \\ \mathrm{H} & -2.421032 & -1.268756 & -0.181393 \\ \mathrm{H} & -1.108018 & -1.299993 & -1.359280 \\ \mathrm{C} & 1.871930 & -0.786120 & -0.733679 \\ \mathrm{C} & 1.925755 & 0.572053 & -0.778691 \\ \mathrm{H} & 1.239427 & -1.344735 & -1.407954 \\ \mathrm{H} & 2.617917 & 1.127112 & -0.163702 \\ \mathrm{H} & 2.518618 & -1.352475 & -0.080593 \\ \mathrm{H} & 1.338856 & 1.132971 & -1.491082 \\ \text { G4 } & \text { Energy= } & & -346.614455 \\ \text { G4 } & \text { Free Energy } & & -346.656579\end{array}$

Epox-TS12b (Optimized at UB3LYP/6-311+G(3df, 2p).
S2=0 Frequencies $--\quad-519.5670$
$\begin{array}{cccc}\text { SCf done: -346.960162 } \\ \text { C } & -0.852173 & 0.043167 & 0.342093 \\ \text { O } & -0.871803 & 0.099579 & 1.660331 \\ \text { O } & 0.634667 & -0.007597 & 0.548866 \\ \text { C } & -1.277008 & 1.309382 & -0.390604 \\ \text { C } & -1.378792 & -1.242453 & -0.282797 \\ \text { H } & -0.802943 & 2.173448 & 0.069953 \\ \text { H } & -0.971092 & -2.099624 & 0.248698 \\ \text { H } & -1.034386 & 1.275831 & -1.454065 \\ \text { H } & -2.358662 & 1.419548 & -0.300890 \\ \text { H } & -2.465250 & -1.259389 & -0.185126 \\ \text { H } & -1.140260 & -1.316939 & -1.345070\end{array}$




$\begin{array}{llrr}\mathrm{C} & 1.941797 & -0.793289 & -0.748262 \\ \mathrm{C} & 1.990253 & 0.568906 & -0.798223 \\ \mathrm{H} & 1.341944 & -1.360375 & -1.443535 \\ \mathrm{H} & 2.656936 & 1.131520 & -0.163250 \\ \mathrm{H} & 2.566555 & -1.353754 & -0.070155 \\ \mathrm{H} & 1.432231 & 1.124478 & -1.536220\end{array}$

\section{TS-13}

S2 $=0.867963$ Frequencies -- -1080.2033 scf done: $\quad-346.776908$

$\begin{array}{lrrr}\mathrm{C} & -1.023681 & 0.012215 & 0.716821 \\ \mathrm{O} & -1.600096 & 0.052330 & 1.986120 \\ \mathrm{O} & 0.275654 & -0.002887 & 1.232778 \\ \mathrm{C} & -1.284624 & 1.261121 & -0.100903 \\ \mathrm{C} & -1.412243 & -1.277534 & 0.017054 \\ \mathrm{H} & -1.000926 & 2.137707 & 0.477632 \\ \mathrm{H} & -1.152567 & -2.123667 & 0.649002 \\ \mathrm{H} & -0.707865 & 1.230923 & -1.024550 \\ \mathrm{H} & -2.345538 & 1.316275 & -0.345609 \\ \mathrm{H} & -2.481871 & -1.289397 & -0.192172 \\ \mathrm{H} & -0.869469 & -1.341959 & -0.925903 \\ \mathrm{C} & 1.927415 & -0.764871 & -1.312579 \\ \mathrm{C} & 2.097474 & 0.538580 & -1.155909 \\ \mathrm{H} & 1.832983 & -1.209811 & -2.295616 \\ \mathrm{H} & 2.197212 & 0.980454 & -0.172393 \\ \mathrm{H} & 1.882482 & -1.432943 & -0.461881 \\ \mathrm{H} & 2.145608 & 1.209853 & -2.004355\end{array}$

G4 Energy=

G4 Free Energy=
$-346.598697$

$-346.648173$ 